\title{
Thermodynamical properties of a noncommutative anti-de Sitter-Einstein-Born-Infeld spacetime from gauge theory of gravity
}

\author{
Román Linares ${ }^{*}$ and Marco Maceda $\odot^{\dagger}$ \\ Departamento de Física Universidad Autónoma Metropolitana-Iztapalapa Av. San Rafael Atlixco 186, \\ C.P. 09340 México City, México \\ Oscar Sánchez-Santos ${ }^{*}$ \\ Universidad Panamericana Augusto Rodin 498, C.P. 03920, México City, México \\ and Instituto de Educación Media Superior de la Ciudad de México Av. División del Norte No. 906, \\ Col. Narvarte Poniente, C. P. 03020 Del. Benito Juárez, México City, México
}

(Received 3 October 2019; accepted 13 January 2020; published 5 February 2020)

\begin{abstract}
We construct a deformed anti-de Sitter-Einstein-Born-Infeld black hole from noncommutative gauge theory of gravity and determine the metric coefficients up to second order on the noncommutative parameter. We analyze the modifications on the thermodynamical properties of the black hole due to the noncommutative contributions, and we show that noncommutativity has as a direct consequence, the removal of critical points.
\end{abstract}

DOI: 10.1103/PhysRevD.101.044008

\section{INTRODUCTION}

In the past years, several approaches have been proposed to explore fundamental interactions in nature at energies beyond those attained at the laboratory. Gauge theories, for example, provide many helpful insights into the nature of particle interactions, and they have been generalized to various contexts. In particular, noncommutative gauge theories using the Seiberg-Witten map [1] have broad applications into the analysis of the scattering process of fundamental particles at high energies [2-6]. On the other hand, loop quantum gravity, string theory, and noncommutative geometry are a few of several approaches that exist aiming to elucidate the structure of spacetime taking into account quantum effects; the link between the string theory and noncommutative geometry has attracted much attention since first noticed [7-13]. The ultimate goal of all these formalisms is to provide a detailed picture of our universe at high energies.

At the Planck scale, noncommutativity manifests itself through discretization of spacetime and quantum gravitational effects are noticeable [14]. A procedure to analysing these effects is to use commutation relations among

\footnotetext{
*lirr@xanum.uam.mx

†mmac@xanum.uam.mx

"osanchezs@up.edu.mx
}

Published by the American Physical Society under the terms of the Creative Commons Attribution 4.0 International license. Further distribution of this work must maintain attribution to the author(s) and the published article's title, journal citation, and DOI. Funded by SCOAP ${ }^{3}$. spacetime coordinates. The implementation of a star product provides a simple realization of these commutation relations and at the same time, generalizes the standard pointwise multiplication of functions. The star product defined on a manifold encodes the quantum structure of spacetime and its discretization naturally.

One of the implications of a noncommutative structure of spacetime is that the standard pointlike nature of particles becomes replaced by a fuzzy picture; objects are no longer localized $[15,16]$. This fundamental difference with respect to the classical scenario leads to the prediction of new effects susceptible of experimental observation in particle physics, gravity and cosmology [17-24]. In all of these scenarios, the fuzzy structure of the sources affects the spacetime with nontrivial consequences.

Furthermore, noncommutative gravity produces quantum corrections and deviations of the classical results that may be used to set bounds on the noncommutative parameter. In particular, the thermodynamical properties of noncommutative spacetimes may be useful in understanding the evolution of black holes when a minimal length scale exists $[25,26]$.

As the Seiberg-Witten map gives a straightforward construction of a noncommutative gauge theory from a commutative one employing a star product, it is natural to ask how this procedure works using the formulation of gravity as a gauge theory. Fortunately enough, gauge theories of gravity have a long history in physics and admit an elegant formulation in the language of fiber bundles [27-30]. These models have as a basis a gauge theory of gravity with de Sitter group as their local symmetry group [31] at the 
classical level. The classical spacetimes obtained in this way are then generalized to the noncommutative framework by a perturbative approach based on the Seiberg-Witten map; such theories of noncommutative gauge-gravity have been formulated and extensively studied in the last years [32-34].

Even if models of pure gravity are exciting per se, either classical or noncommutative, the general situation where other interactions are present gives valuable information on several classes of phenomena. For example, in active galaxy nuclei, if an electromagnetic field exists, it should interact with itself in a nonlinear way, and the motion of charged test particles provides a way to quantify differences in comparison when the nonlinearity is absent.

It is then necessary for a complete picture of fundamental interactions to include at some point, nonlinear electrodynamics. In this regard, Born-Infeld (BI) electrodynamics $[35,36]$ and the corresponding gravitational solution, the standard Einstein-Born-Infeld (EBI) metric [37], have a broad variety of applications and extensions, from inflation and branes to AdS and dilaton black holes. For these reasons, in this paper, we use the gauge formulation of gravity to construct a noncommutative anti-de SitterEinstein-Born-Infeld (AdSEBI) black hole. A thermodynamical analysis of the solutions, based on the equation of state and Gibbs function, may reveal the existence or not of new critical points and new phase transitions induced by noncommutativity.

The paper is organized as follows: In Sec. II, we review the formalism of gauge theory of gravity based on the Sitter group, and use this approach there to solve explicitly the field equations for the specific case of AdSEBI spacetime; we recover the well-known EBIon solution with a cosmological constant when the BI parameter vanishes. We discuss then the deformation technique of gauge-gravity theory based on the Seiberg-Witten map and apply it to the commutative AdSEBI black hole in Sec. III to define the noncommutative counterpart. The analysis of the noncommutative effects on the thermodynamical properties of the AdSEBI black hole is discussed in Sec. IV; there we show that critical points may be removed. Finally, in the Conclusions section, we give some remarks and perspectives about future work.

\section{GAUGE THEORY OF GRAVITY}

We review briefly the main ingredients of gauge theory of gravity with de Sitter group as a local symmetry [31,38,39]. The starting point is a 4-dimensional Minkowski metric in spherical coordinates

$$
d s^{2}=d t^{2}-d r^{2}-r^{2}\left(d \theta^{2}+\sin ^{2} \theta d \phi^{2}\right) .
$$

This spacetime is the base manifold where the gravitational field will be described by gauge field potentials $h_{\mu}^{A}$, $\mu=0, \ldots, 3, A=1, \ldots, 10$. The gauge potentials depend on the coordinates of the base manifold and are split into two sets: four tetrads $e^{a}$ with components $e_{\mu}^{a}(x)$, and six spin connections with components $\omega_{\mu}^{a b}(x)$; the latter possess the standard skew property $\omega_{\mu}^{a b}(x)=-\omega_{\mu}^{b a}(x)$.

Using the tetrad and the spin connections, we define the following antisymmetric strength tensors

$$
\begin{aligned}
F_{\mu \nu}^{a}:= & \partial_{\mu} e_{\nu}^{a}-\partial_{\nu} e_{\mu}^{a}+\left(\omega_{\mu}^{a b} e_{\nu}^{c}-\omega_{\nu}^{a b} e_{\mu}^{c}\right) \eta_{b c}, \\
F_{\mu \nu}^{a b}:= & \partial_{\mu} \omega_{\nu}^{a b}-\partial_{\nu} \omega_{\mu}^{a b}+\left(\omega_{\mu}^{a c} \omega_{\nu}^{d b}-\omega_{\nu}^{a c} \omega_{\mu}^{d b}\right) \eta_{b c} \\
& +4 \lambda^{2}\left(e_{\mu}^{b} e_{\nu}^{a}-e_{\mu}^{a} e_{\nu}^{b}\right) .
\end{aligned}
$$

The action associated with these gauge gravitational fields is

$$
S_{g}=\frac{1}{16 \pi G} \int d^{4} x e F,
$$

where $e:=\operatorname{det}\left(e_{\mu}^{a}\right)$ and

$$
F:=F_{\mu \nu}^{a b} \bar{e}_{a}^{\mu} \bar{e}_{b}^{\nu} .
$$

Here the elements $\bar{e}_{a}^{\mu}(x)$ are the inverse elements of the tetrad components $e_{\mu}^{a}(x)$, i.e., $\bar{e}_{a}^{\mu} \bar{e}_{\nu}^{a}=\delta_{\nu}^{\mu}$, etc.

As we are dealing with gauge gravitational fields, an electromagnetic gravitational potential $A_{\mu}(x)$ is also created; its corresponding action is given by

$$
S_{e m}=-\frac{1}{4 K g^{2}} \int d^{4} x e A_{\mu}^{a} \bar{A}_{a}^{\mu},
$$

where the quantities $A_{\mu}^{a}$ and $\bar{A}_{a}^{\mu}$ are defined as

$A_{\mu}^{a}:=A_{\mu}^{\nu} e_{\nu}^{a}, \quad A_{\mu}^{\nu}:=\bar{e}_{a}^{\nu} \bar{e}_{b}^{\rho} \eta^{a b} A_{\mu \rho}, \quad \bar{A}_{a}^{\mu}:=A_{\nu}^{\mu} \bar{e}_{a}^{\nu}$,

in terms of the electromagnetic field tensor

$$
A_{\mu \nu}:=\partial_{\mu} A_{\nu}-\partial_{\nu} A_{\mu} .
$$

In the above expressions, $g$ represents a coupling constant and $K$ is a normalization constant that will be chosen later to simplify certain expressions.

The full action of the model takes into account the gauge fields and the electromagnetic field

$$
S=\int d^{4} x e\left[\frac{1}{16 \pi G} F-\frac{1}{4 K g^{2}} A_{\mu}^{a} \bar{A}_{a}^{\mu}\right]
$$

Variation of this action with respect to the tetrad components $e_{\mu}^{a}(x)$ gives the following field equations

$$
F_{\mu}^{a}-\frac{1}{2} F e_{\mu}^{a}=8 \pi G T_{\mu}^{a},
$$

where $F_{\mu}^{a}:=F_{\mu \nu}^{a b} \bar{e}_{b}^{\nu}$ and the energy-momentum tensor is given by 


$$
T_{\mu}^{a}=\frac{1}{K g^{2}}\left(A_{\mu}^{b} A_{\nu}^{a} \bar{e}_{b}^{\nu}-\frac{1}{4} A_{\nu}^{b} A_{b}^{\nu} e_{\mu}^{a}\right)
$$

When considering additional fields interacting with gravity, the corresponding energy-momentum tensors should be included on the right-hand side of Eq. (10). On the other hand, the field equations for the gravitational gauge potentials $\omega_{\mu}^{a b}(x)$ are

$$
F_{\mu \nu}^{a}=0 .
$$

Physically, this condition amounts to the absence of torsion in the theory as it is the situation in general relativity.

\section{A. Commutative AdSEBI spacetime from gauge theory of gravity}

To illustrate the previous formalism of gauge-gravity [31,40-42] for classical spacetimes, we use it now to obtain a commutative anti-de Sitter-Einstein-Born-Infeld black hole solution. The starting point is a gravitational gauge field with spherical symmetry given by the following Ansatz

$$
\begin{array}{ll}
e_{\mu}^{0}=(A, 0,0,0), & e_{\mu}^{1}=\left(0, \frac{1}{A}, 0,0\right), \\
e_{\mu}^{2}=(0,0, r, 0), & e_{\mu}^{3}=(0,0,0, r \sin \theta),
\end{array}
$$

together with the spin connections

$$
\omega_{\mu}^{01}=(U, 0,0,0), \quad \omega_{\mu}^{02}=\omega_{\mu}^{03}=0, \quad \omega_{\mu}^{12}=(0,0, A, 0),
$$$$
\omega_{\mu}^{13}=(0,0,0, A \sin \theta), \quad \omega_{\mu}^{12}=(0,0,0, \cos \theta),
$$

where $A$ and $U$ are functions of the radial coordinate $r$. On the other hand, the density Lagrangian of the BI electrodynamics is chosen as

$$
L_{B I}=\frac{2}{b^{2}}\left(1-\sqrt{1+b^{2} F_{\mu \nu} F^{\mu \nu}}\right),
$$

where $b$ is the BI parameter; in the limit when $b \rightarrow 0$, we recover standard Maxwell's electrodynamics. From the above Lagrangian, we obtain straightforwardly the components of the energy-momentum tensor of the BI electrodynamics. In consequence, the field equations for the tetrad elements $e_{\mu}^{a}$ as deduced from Eqs. (10) including the BI electromagnetic contributions are

$$
\begin{aligned}
& \left(\frac{-2 r A A^{\prime}+1-A^{2}+12 \lambda^{2} r^{2}}{r^{2}}\right) A=\left(\frac{\sqrt{r^{4}+b^{2} Q^{2}}}{r^{2} b^{2}}-\frac{1}{b^{2}}\right) A, \\
& \left(\frac{2 r U+1-A^{2}+12 \lambda^{2} r^{2}}{r^{2}}\right) \frac{1}{A}=\left(\frac{\sqrt{r^{4}+b^{2} Q^{2}}}{r^{2} b^{2}}-\frac{1}{b^{2}}\right) \frac{1}{A},
\end{aligned}
$$

$$
\begin{gathered}
r U^{\prime}+U-A A^{\prime}+12 \lambda^{2} r=\frac{r}{b^{2}}\left(\frac{r^{2}}{\sqrt{r^{4}+b^{2} Q^{2}}}-1\right), \\
\left(\frac{r^{2} U^{\prime}+r U-r A A^{\prime}+12 \lambda^{2} r^{2}}{r^{2}}\right) r \sin \theta \\
=\frac{r \sin \theta}{b^{2}}\left(\frac{r^{2}}{\sqrt{r^{4}+b^{2} Q^{2}}}-1\right) .
\end{gathered}
$$

We see that Eqs. (18) and (19) are the same field equation. We also notice that Eqs. (16) and (17) become the same field equation if $U=-A A^{\prime}=-\left(A^{2} / 2\right)^{\prime}$; this last relation is also a consequence from Eq. (12). Therefore, the relevant field equations to solve are

$$
\begin{aligned}
& -\frac{2 A A^{\prime}}{r}+\frac{1-A^{2}}{r^{2}}+12 \lambda^{2}=-\frac{1}{b^{2}}+\frac{\sqrt{r^{4}+b^{2} Q^{2}}}{r^{2} b^{2}}, \\
& -\frac{1}{2}\left(A^{2}\right)^{\prime \prime}-\frac{2 A A^{\prime}}{r}+12 \lambda^{2}=-\frac{1}{b^{2}}+\frac{r^{2}}{b^{2} \sqrt{r^{4}+b^{2} Q^{2}}} .
\end{aligned}
$$

If we now take the difference between Eqs. (20) and (21), we obtain the single equation

$$
\frac{1}{2}\left(A^{2}\right)^{\prime \prime}+\frac{1-A^{2}}{r^{2}}=\frac{Q^{2}}{r^{2} \sqrt{r^{4}+b^{2} Q^{2}}},
$$

or equivalently

$$
r^{2}\left(A^{2}\right)^{\prime \prime}-2 A^{2}+2=\frac{2 Q^{2}}{\sqrt{r^{4}+b^{2} Q^{2}}} .
$$

The solution to Eq. (23) is

$$
\begin{aligned}
A^{2}= & 1-\frac{2 M}{r}-\frac{\Lambda}{3} r^{2}+\frac{2}{3} \frac{r^{2}}{b^{2}}\left(1-\sqrt{1+\frac{r_{0}^{4}}{r^{4}}}\right) \\
& +\frac{4}{3} \frac{r_{0}^{4}}{b^{2} r^{2}} F_{1}\left(\frac{1}{4}, \frac{1}{2} ; \frac{5}{4} ;-\frac{r_{0}^{4}}{r^{4}}\right)
\end{aligned}
$$

where we have set $r_{0}^{4}:=b^{2} Q^{2}$ and $\Lambda:=-12 \lambda^{2}$. In the limit $b \rightarrow 0$, we recover the black hole solution of the model studied in [33].

\section{NONCOMMUTATIVE ADSEBI BLACK HOLE FROM GAUGE THEORY OF GRAVITY}

Having as starting point a gauge theory of gravity, a natural generalization of this theory to the noncommutative framework makes use of the Seiberg-Witten map [1]. A deformation based on the Moyal-Groenewold star product $[43,44]$ makes use of constant noncommutative parameters 
$\Theta^{\mu \nu}$; they define the commutation relations among the spacetime coordinates providing the noncommutative structure of spacetime as

$$
\left[x^{\mu}, x^{\nu}\right]=i \Theta^{\mu \nu},
$$

where $\Theta^{\mu \nu}$ is an antisymmetric matrix. It follows then that the product of any two fields is given by the Moyal star product

$$
(f \star g)(x)=f(x) e^{\frac{1}{2} \Theta^{\mu \nu} \tilde{\partial}_{\mu} \vec{\partial}_{\nu}} g(x) .
$$

Along the lines of [32], noncommutative corrections to commutative quantities are obtained as follows. First, the noncommutative gauge gravity fields $\hat{\omega}_{\mu}^{A B}(x, \Theta)$ are subject to the reality conditions

$$
\begin{aligned}
\omega_{\mu}^{\hat{A} B_{\mu}^{\dagger}}(x, \Theta) & =-\hat{\omega}_{\mu}^{B A}(x, \Theta), \\
\omega_{\mu}^{\hat{A} B_{\mu}^{\dagger}}(x, \Theta)^{r} & =\hat{\omega}_{\mu}^{A B}(x,-\Theta)=-\hat{\omega}_{\mu}^{B A}(x, \Theta),
\end{aligned}
$$

where $\dagger$ denotes complex conjugation; these conditions guarantee that the noncommutative gauge fields are real. Then, we expand the fields $\omega^{\hat{A} B_{\mu}^{\dagger}}(x, \Theta)$ in powers of the noncommutative parameter $\Theta$,

$$
\begin{aligned}
\omega_{\mu}^{\hat{A} B^{+}}(x, \Theta)= & \omega_{\mu}^{A B}(x, \Theta)-i \Theta^{\nu \rho} \omega_{\mu \nu \rho}^{A B}(x) \\
& +\Theta^{\nu \rho} \Theta^{\lambda \tau} \omega_{\mu \nu \rho \lambda \tau}^{A B}(x)+\cdots
\end{aligned}
$$

The reality conditions, Eqs. (27), after expansion become

$$
\begin{aligned}
\omega_{\mu}^{A B}(x) & =-\omega_{\mu}^{B A}(x), \quad \omega_{\mu \nu \rho}^{A B}(x)=\omega_{\mu \nu \rho}^{B A}(x), \\
\omega_{\mu \nu \rho \lambda \tau}^{A B}(x) & =-\omega_{\mu \nu \rho \lambda \tau}^{B A}(x) .
\end{aligned}
$$

Using now the Seiberg-Witten map, we obtain straightforwardly the corrections up to second order; they are [32]

$$
\begin{aligned}
\omega_{\mu \nu \rho}^{A B}(x)= & \frac{1}{4}\left\{\omega_{\nu}, \partial_{\rho} \omega_{\mu} F_{\mu \nu}\right\}^{A B}, \\
\omega_{\mu \nu \rho \lambda \tau}^{A B}(x)= & \frac{1}{32}\left(-\left\{\omega_{\lambda}, \partial_{\tau}\left\{\omega_{\nu}, \partial_{\rho} \omega_{\mu} F_{\mu \nu}\right\}\right\}\right. \\
& +2\left\{\omega_{\lambda},\left\{F_{\tau \nu}, F_{\mu \rho}\right\}\right\} \\
& -\left\{\omega_{\lambda},\left\{\omega_{\nu}, D_{\rho} F_{\mu \nu}+\partial_{\rho} F_{\tau \mu}\right\}\right\} \\
& -\left\{\left\{\omega_{\nu}, \partial_{\rho} \omega_{\mu}+F_{\mu \lambda}\right\},\left(\partial_{\tau} \omega_{\mu}+F_{\tau \mu}\right)\right\} \\
& \left.+2\left[\partial_{\nu} \omega_{\lambda}, \partial_{\rho}\left(\partial_{\tau} \omega_{\mu}+F_{\tau \mu}\right)\right]\right)^{A B},
\end{aligned}
$$

where we use the following definitions

$$
\begin{aligned}
\{\alpha, \beta\}^{A B} & :=\alpha^{A C} \beta_{C}^{B}+\beta^{A C} \alpha_{C}^{B}, \\
{[\alpha, \beta]^{A B} } & :=\alpha^{A C} \beta_{C}^{B}-\beta^{A C} \alpha_{C}^{B},
\end{aligned}
$$

and

$$
D_{\mu} F_{\rho \sigma}^{A B}:=\partial_{\mu} F_{\rho \sigma}^{A B}+\left(\omega_{\mu}^{A C} F_{\rho \sigma}^{B D}+\omega_{\mu}^{B C} F_{\rho \sigma}^{D A}\right) \eta_{C D} .
$$

Notice that we only need to know the classical quantities to determine the noncommutative contributions to them. On the other hand, the noncommutative corrections up to second order in the noncommutativity parameter to the classical tetrad fields are [45-47]

$$
\hat{e}_{\mu}^{a}(x, \Theta)=e_{\mu}^{a}(x)-i \Theta^{\nu \rho} e_{\mu \nu \rho}^{a}(x)+\Theta^{\nu \rho} \Theta^{\lambda \tau} e_{\mu \nu \rho \lambda \tau}^{a}(x),
$$

where

$$
e_{\mu \nu \rho}^{a}(x)=\frac{1}{4}\left[\omega_{\mu}^{a c} \partial_{\rho} e_{\mu}^{d}+\left(\partial_{\rho} \omega_{\mu}^{a c}+F_{\rho \mu}^{a c}\right) e_{\nu}^{d}\right] \eta_{c d},
$$

and

$$
\begin{aligned}
e_{\mu \nu \rho \lambda \tau}^{a}(x)= & \frac{1}{32}\left[2\left\{F_{\tau \nu}, F_{\mu \rho}\right\}^{a b} e_{\lambda}^{c}-\omega_{\lambda}^{a b}\left(D_{\rho} F_{\tau \mu}^{c d}+\partial_{\rho} F_{\tau \mu}^{c d}\right) e_{\nu}^{m} \eta_{d m}-\left\{\omega_{\nu},\left(D_{\rho} F_{\tau \mu}+\partial_{\rho} F_{\tau \mu}\right)\right\}^{a b} e_{\lambda}^{c}+\partial_{\nu} \omega_{\lambda}^{a b} \partial_{\rho} \partial_{\tau} e_{\mu}^{c}\right. \\
& -\partial_{\tau}\left\{\omega_{\nu},\left(\partial_{\rho} \omega_{\mu}+F_{\rho \mu}\right)\right\}^{a b} e_{\lambda}^{c}-\omega_{\lambda}^{a b} \partial_{\tau}\left(\omega_{\nu}^{c d} \partial_{\rho} e_{\mu}^{m}+\left(\partial_{\rho} \omega_{\mu}^{c d}+F_{\rho \mu}^{c v}\right) e_{\mu}^{m}\right) \eta_{d m}-2 \partial_{\rho}\left(\partial_{\tau} \omega_{\nu}^{a b}+F_{\tau \mu}^{a b}\right) \partial_{\nu} e_{\lambda}^{c} \\
& \left.-\left\{\omega_{\nu},\left(\partial_{\rho} \omega_{\lambda}+F_{\rho \lambda}\right)\right\}^{a b} \partial_{\tau} e_{\mu}^{c}-\left(\partial_{\tau} \omega_{\mu}^{a b}+F_{\tau \mu}^{a b}\right)\left(\omega_{\nu}^{c d} \partial_{\rho} e_{\mu}^{m}+\left(\partial_{\tau} \omega_{\mu}^{a b}+F_{\tau \mu}^{a b}\right) e_{\mu}^{m} \eta_{d m}\right)\right] \eta_{b c} .
\end{aligned}
$$

The noncommutative metric can then be found from the expression [32]

$$
\hat{g}_{\mu \nu}=\frac{1}{2} \eta_{a b}\left(\hat{e}_{\mu}^{a} \star \hat{e}^{b \dagger}{ }_{\nu}+\hat{e}_{\mu}^{b} \star \hat{e}^{a \dagger}{ }_{\nu}\right),
$$

where $\dagger$ denotes complex conjugation.

We now proceed to calculate the noncommutative metric for the EBI spacetime using the formalism just described; using Eqs. (34) and (35) together with Eqs. (13) and (14), we obtain the following expressions 


$$
\begin{aligned}
& \hat{g}_{11}=\frac{1}{A^{2}}+\frac{1}{4} \frac{A^{\prime \prime}}{A} \Theta^{2}+\mathcal{O}\left(\Theta^{4}\right), \\
& \hat{g}_{22}=r^{2}+\frac{1}{16}\left(A^{2}+11 r A A^{\prime}+16 r^{2} A^{\prime 2}+12 r^{2} A A^{\prime \prime}\right) \Theta^{2}+\mathcal{O}\left(\Theta^{4}\right), \\
& \hat{g}_{33}=r^{2} \sin ^{2} \theta+\frac{1}{16}\left[4\left(2 r A A^{\prime}-r \frac{A^{\prime}}{A}+r^{2} A A^{\prime \prime}+r^{2} A^{\prime 2}\right) \sin ^{2} \theta+\cos ^{2} \theta\right] \Theta^{2}+\mathcal{O}\left(\Theta^{4}\right), \\
& \hat{g}_{00}=-A^{2}-\frac{1}{4}\left(2 r A A^{\prime 3}+r A^{3} A^{\prime \prime \prime}+A^{3} A^{\prime \prime}+r^{2} A^{\prime 2}+2 A^{2} A^{\prime 2}+5 r A^{2} A^{\prime} A^{\prime \prime}\right) \Theta^{2}+\mathcal{O}\left(\Theta^{4}\right),
\end{aligned}
$$

up to second order on the noncommutative parameter. In the above expressions, the function $A$ is given by Eq. (24); we also set $\Theta^{12}=-\Theta^{21}=: \Theta$ as the only nonvanishing values for the noncommutative parameters. We recover the classical result for the components of the metric in the commutative limit $\Theta \rightarrow 0$.

Using now the explicit expression for $A$, a straightforward calculation shows then that the coefficient $\hat{g}_{00}$ of the noncommutative metric tensor is

$$
\begin{aligned}
\hat{g}_{00}= & 1-\frac{2 M}{r}-\frac{\Lambda r^{2}}{3}+\frac{2}{3} \frac{r^{2}}{b^{2}}\left(1-\sqrt{1+\frac{b^{2} Q^{2}}{r^{4}}}\right)+\frac{4 Q^{2} f(r)}{3 r}+\frac{1}{16}\left\{2 r \left[\frac{4}{3 b^{2}}-\frac{4 M}{r^{3}}-\frac{4 r^{4}-8 b^{2} Q^{2}}{3 b^{2} r^{2} \sqrt{r^{4}+b^{2} Q^{2}}}\right.\right. \\
& \left.-\frac{2 \Lambda}{3}+\frac{8 Q^{2} f(r)}{3 r^{3}}\right]\left[\frac{2 M}{r^{2}}+\frac{4 r}{3 b^{2}}\left(1-\sqrt{1+\frac{b^{2} Q^{2}}{r^{4}}}\right)-\frac{2 \Lambda r}{3}-\frac{4 Q^{2} f(r)}{3 r^{2}}\right] \\
& +\left[\frac{2 M}{r^{2}}+\frac{4 r}{3 b^{2}}\left(1-\sqrt{1+\frac{b^{2} Q^{2}}{r^{4}}}\right)-\frac{\Lambda r^{2}}{3}-\frac{4 Q^{2} f(r)}{3 r^{2}}\right]^{2}+2\left[1-\frac{2 M}{r}+\frac{2}{3} \frac{r^{2}}{b^{2}}\left(1-\sqrt{1+\frac{b^{2} Q^{2}}{r^{4}}}\right)\right. \\
& \left.\left.-\frac{\Lambda r^{2}}{3}+\frac{4 Q^{2} f(r)}{3 r}\right]\left[\frac{4}{3 b^{2}}+\frac{8 M}{r^{3}}-\frac{4\left(4 b^{4} Q^{4}+11 b^{2} Q^{2} r^{4}+r^{8}\right)}{3 b^{2} r^{2}\left(r^{4}+b^{2} Q^{2}\right)^{3 / 2}}-\frac{2 \Lambda}{3}-\frac{16 Q^{2} f(r)}{3 r^{3}}\right]\right\} \Theta^{2}+\mathcal{O}\left(\Theta^{4}\right) .
\end{aligned}
$$

where

$$
f(r):=\frac{1}{2 \sqrt{b Q}} F\left[\arccos \left\{\frac{r^{2}+b Q}{r^{2}+b Q}\right\}, \frac{1}{\sqrt{2}}\right]=\frac{1}{2 r_{2}} F_{1}\left(\frac{1}{4}, \frac{1}{2}, \frac{5}{4},-\frac{b^{2} Q^{2}}{r^{4}}\right)
$$

The explicit form of the remaining metric coefficients can be determined in a similar way. Once they are known, several quantities may be evaluated. In particular, using the above expression for $\hat{g}_{00}$, we can determine the presence or absence of horizons. In Fig. 1, we plot $\hat{g}_{00}$ as a function of $r$; for large values of $r$, we recover the commutative behavior of the metric, while the short distance behavior changes. We see that the noncommutative AdSEBI black
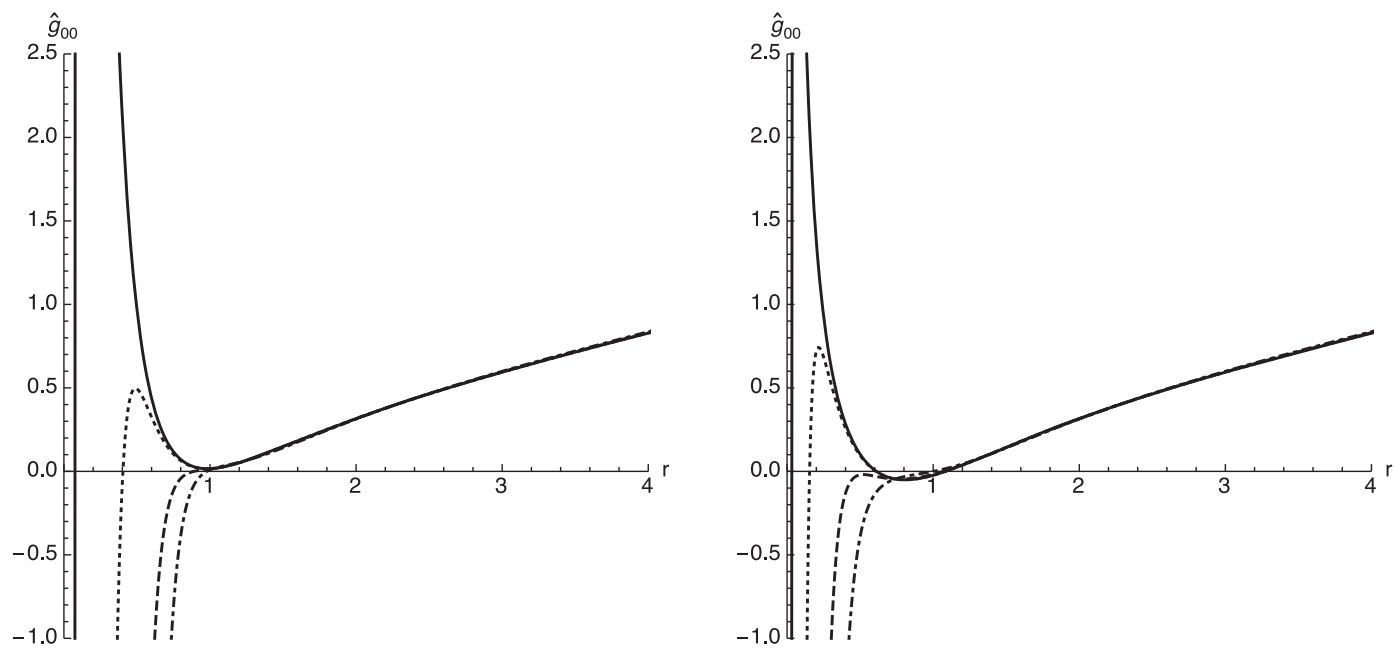

FIG. 1. Plot of $\hat{g}_{00}$ as a function of the radial variable $r$ for $\Theta=0.01,0.1,0.4,0.8$ (solid, dotted, dashed, dot-dashed line) with $Q=1, \Lambda=-1 / 20, M=1$ with $b=0.1$ (left panel) or $b=1$ (right panel). The noncommutative AdSEBI black hole can develop two or more horizons for some values of the BI parameter $b$; they eventually collapse to only one as $\theta$ increases. 
hole may develop up to three horizons for some values of its parameters; we also notice that as noncommutative effects increase, an extremal black hole may arise with a single horizon.

\section{THERMODYNAMICS}

The fact that a thermodynamical approach can be used to study black hole solutions is remarkable since it leads to a notion of temperature based on the surface gravity of the black hole. Quantum effects can also be incorporated in this picture, providing a more in-depth insight into certain aspects expected to exist in a quantum theory of gravity.

In the following, we use the results of the previous sections to study the thermodynamics of the noncommutative AdSEBI black hole from gauge-gravity; the resulting expressions are direct generalizations of classical results. The first step in this direction is the calculation of the ADM mass. Using standard perturbation theory, the ADM mass up to second order in the noncommutative parameter is

$$
\begin{aligned}
M_{\mathrm{ADM}}= & \frac{r_{H}}{2}-\frac{\Lambda r_{H}^{3}}{6}+\frac{r_{H}^{3}}{3 b^{2}}\left(1-\sqrt{1+\frac{b^{2} Q^{2}}{r_{H}^{4}}}\right)+\frac{4 Q^{2} f\left(r_{H}\right)}{3 r_{H}}-\frac{r_{H}^{2}}{64}\left[\frac{4}{r_{H}}\left(\frac{2 Q^{2}}{\sqrt{r_{H}^{4}+b^{2} Q^{2}}}-1\right)\right. \\
& \left.\times\left(\frac{1}{r_{H}}+\frac{2 r_{H}}{b^{2}}\left(1-\sqrt{1+\frac{b^{2} Q^{2}}{r_{H}^{4}}}\right)-\Lambda r_{H}\right)+\left(\frac{1}{r_{H}}+\frac{2 r_{H}}{b^{2}}\left(1-\sqrt{1+\frac{b^{2} Q^{2}}{r_{H}^{4}}}\right)-\Lambda r_{H}\right)^{2}\right] \Theta^{2}+\mathcal{O}\left(\Theta^{4}\right),
\end{aligned}
$$

where $r_{H}$ is the radius of the outer horizon. We see that the well-known classical result gives the leading order in this expression. On the other hand, the Hawking temperature becomes

$$
\begin{aligned}
T_{H}= & \left.\frac{1}{4 \pi} \frac{d \hat{g}_{00}}{d r}\right|_{r_{H}} \\
= & \frac{1}{4 \pi r_{H}}\left[1+\frac{2 r_{H}^{2}}{b^{2}}-\Lambda r_{H}^{2}+\frac{2 r_{H}^{2}}{b^{2}}\left(1-\sqrt{1+\frac{b^{2} Q^{2}}{r_{H}^{4}}}\right)\right] \\
& +F_{2}(r) \Theta^{2}+\mathcal{O}\left(\Theta^{4}\right),
\end{aligned}
$$

where the explicit expression for the function $F_{2}(r)$, rather cumbersome, is provided in the Appendix. We expect that

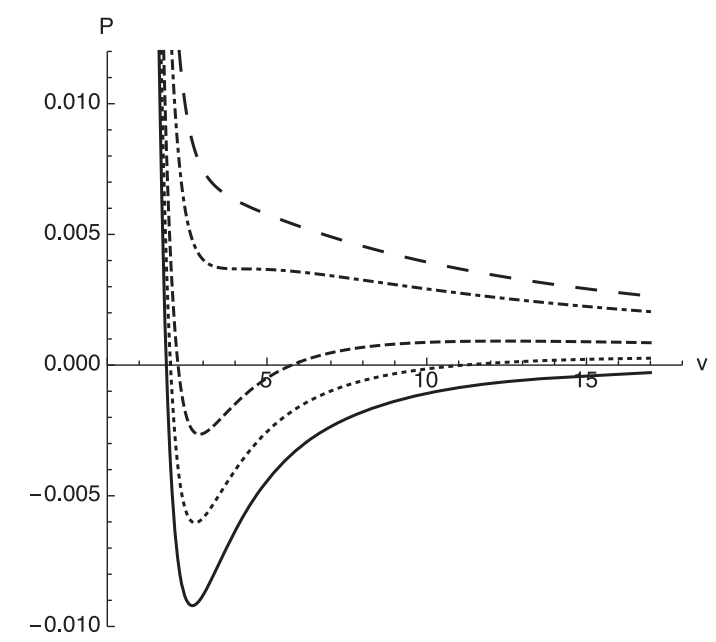

increasing orders in perturbation theory will give us more involved expressions for the noncommutative corrections.

We can also find the corrections to the pressure up to second order in the noncommutative parameter. Using the standard definition $P:=-\Lambda / 8 \pi$, we have the result

$$
\begin{aligned}
P= & \frac{T_{H}}{v}-\frac{1}{2 \pi v^{2}}+\frac{b^{2}}{4 \pi}\left(1-\sqrt{1+\frac{16 b^{2} Q^{2}}{v^{4}}}\right) \\
& +F_{3}(r) \Theta^{2}+\mathcal{O}\left(\Theta^{4}\right),
\end{aligned}
$$

where the specific volume is $v:=2 r_{H}$, and the explicit expression for the function $F_{3}(r)$ can be found in the Appendix. In Fig. 2, we show several isotherms associated

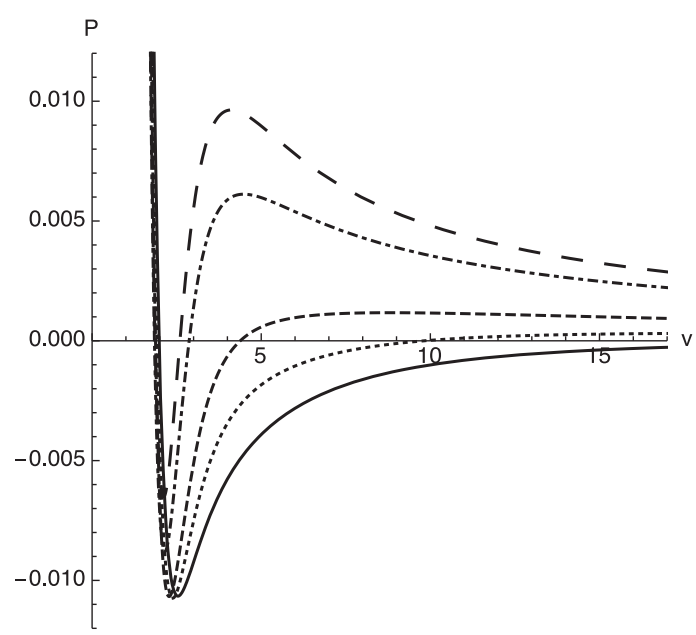

FIG. 2. $P-v$ diagram of noncommutative AdSEBI black hole. The corresponding values for the temperature are $T=0.0043$, 0.04362, 0.02362, 0.01362, and 0.05362 (solid, dotted, dashed, dot-dashed, and long dashed line) with $b=1, Q=1$ in both plots. For the plot in the left panel, $\Theta=0.2$ and for the plot in the right panel $\Theta=0.6$; noncommutativity makes harder the presence of critical points. 


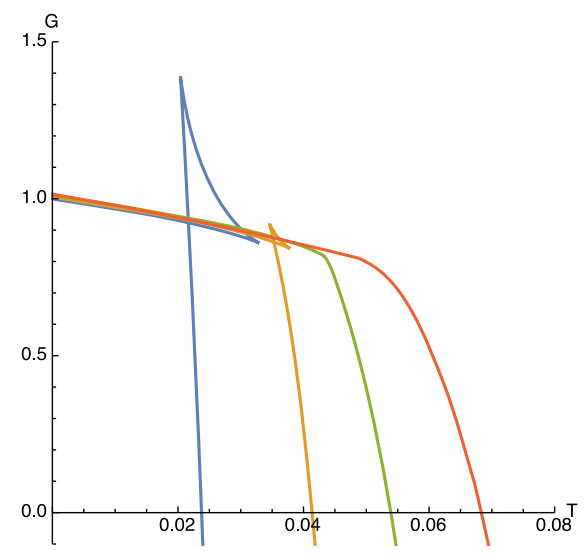

(a)

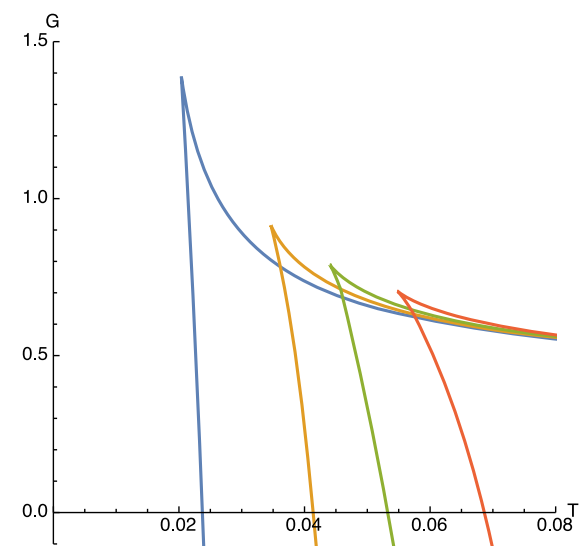

(d)

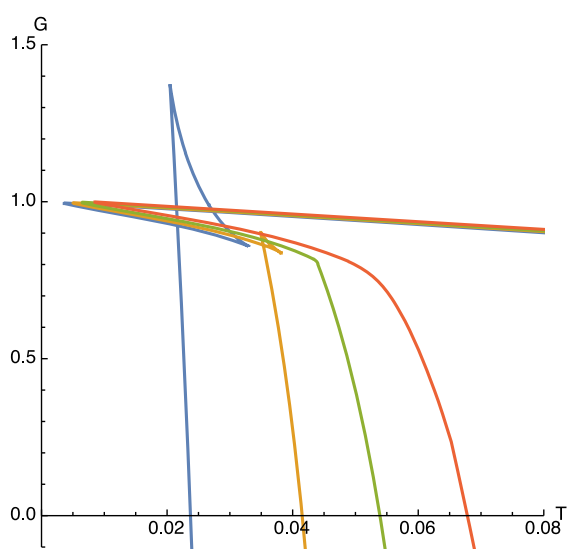

(b)

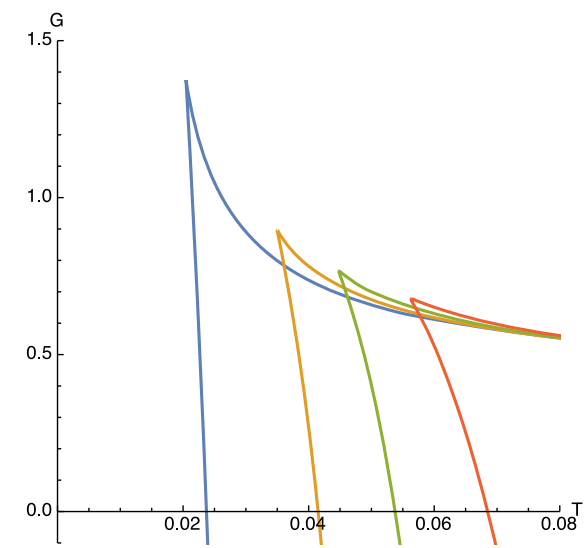

(e)

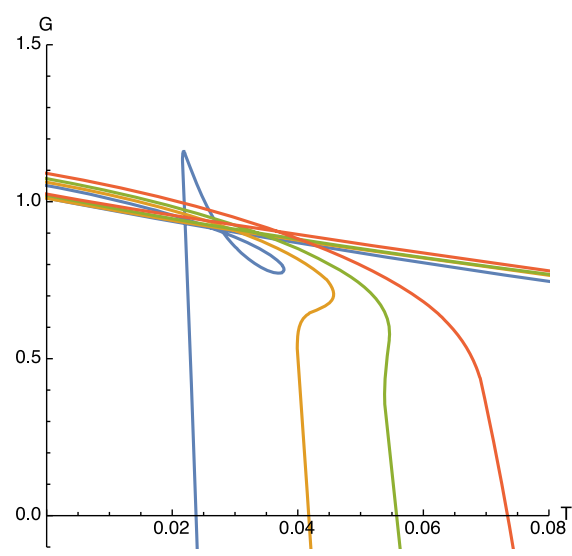

(c)

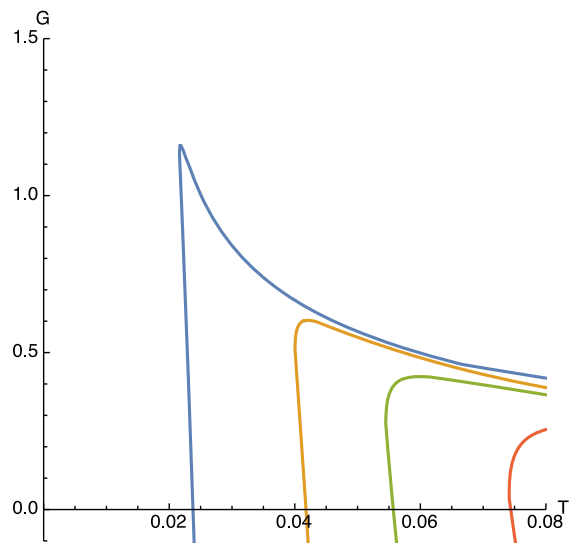

(f)

FIG. 3. The plots show the Gibbs function for different values of the noncommutative parameter $\theta$ and the BI parameter $b$ : in the upper row $b=0.001$ and $\Theta=0,0.1,0.4$ from left to right, in the lower row $b=10$ and $\Theta=0,0.1,0.4$ from left to right. In each graph the pressure $p$ takes the values $96 \pi P=0.2,0.6,1,1.6$ and $Q=1$. Classical $\mathrm{RN}$ and Schwarzschild behaviors are shown in graphs 3(a) and 3(d) respectively.

with the above equation of state; they have the particular form of isothermals of the van der Waals equation. For a fixed value of the BI parameter $b$, it is clear that for a small value of the noncommutative parameter $\theta$, a critical point exists, meanwhile for a larger value of $\theta$, the critical point is absent. Noncommutative effects make harder the presence of critical points.

Besides the analysis of the equation of state of the noncommutative AdSEBI black hole, a discussion of critical points is achieved by calculating the corresponding Gibbs function $G$. In the commutative cases, both Schwarzschild and RN spacetimes have points in the $G-T_{H}$ plane where the derivative of $G$ as a function of the temperature $T_{H}$ is ill defined, leading to the existence of phase transitions.

To analyze the existence of phase transitions in the noncommutative AdSEBI black hole solution, we plot in Fig. 3 the noncommutative Gibbs function

$$
G=M_{A D M}-T_{H} S,
$$

for several values of the noncommutative parameter $\Theta$. The explicit expression for $G$ can be obtained by using Eq. (40) together with Eq. (41) and $S:=\pi r_{H}^{2}$. The BI parameter $b$ has a nontrivial effect on the derivatives of $G$ in the commutative case, allowing the transition from Schwarzschild to RN spacetimes, and we have the standard graphs for the Gibbs function of those two situations. When $\Theta$ is not vanishing and increasing, noncommutativity manifest, and we see clearly that the curves become smoother. Eventually, the derivatives of $G$ become singlevalued for $\Theta=0.4$; a phase transition is no longer present.

\section{CONCLUSIONS}

Using a noncommutative gauge theory of gravity based on the Seiberg-Witten map, we have constructed a noncommutative model of gravity coupled to the nonlinear BI electrodynamics. We calculated the metric coefficients associated with the AdSEBI spacetime explicitly up to second order on the noncommutative parameter using noncommutative gauge gravity fields. This approach is 

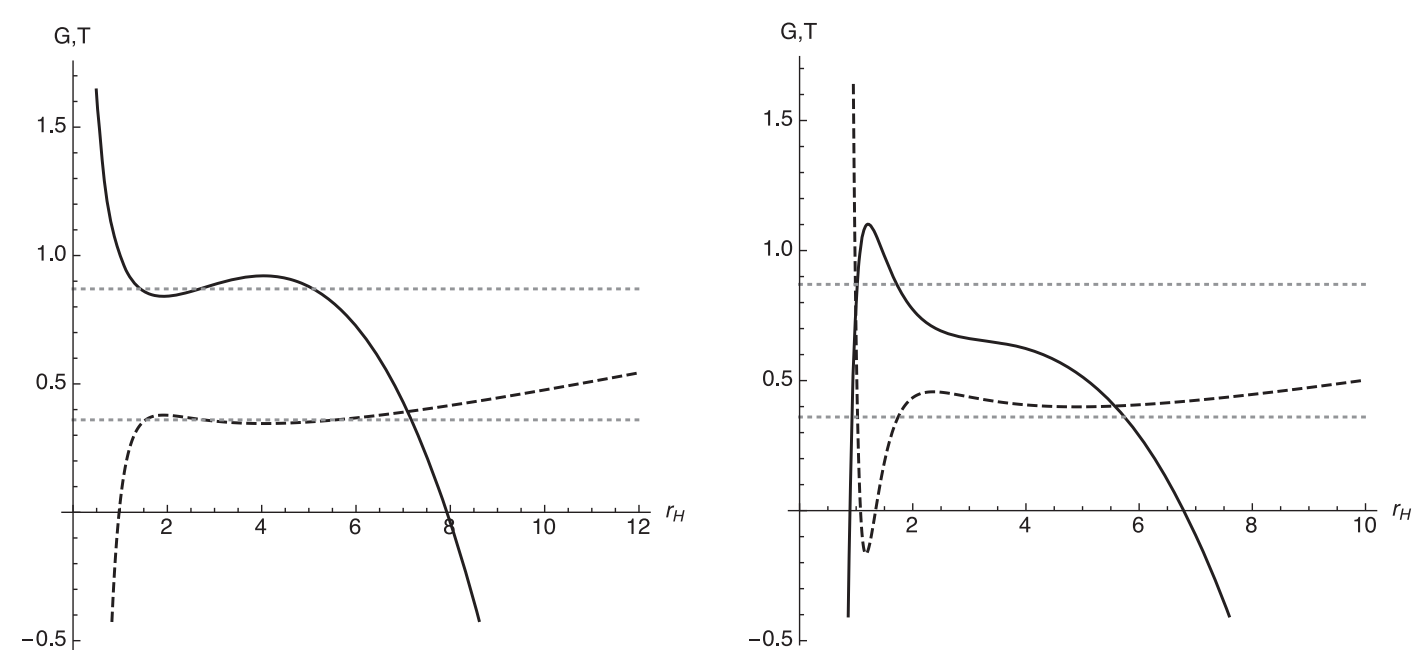

FIG. 4. Behavior of the Gibbs function $G$ (solid line) and the temperature $T$ (dashed line) of the black hole as a function of its horizon radius $r_{H}$; notice the degeneracy associated to the commutative case (left panel with $\theta=0$ ) for a given value of $G$. When the noncommutative parameter is turned on, the degeneracy is partially lifted (right panel with $\theta=0.4$ ). In these plots, we set $b=$ $0.1,96 \pi P=0.6, Q=1$ and the temperature $T$ was scaled by a factor of ten to fit in the same graph.

perturbative, the lowest order being the classical AdSEBI spacetime, and it allows the incorporation of noncommutative effects into standard results. Furthermore, quantities like the ADM mass and the Hawking temperature of the AdSEBI black hole solution allowed quick calculations. Besides this feature, the analysis of thermodynamical properties was also straightforward.

For a given temperature of a black hole, it is possible to have two different horizon radii leading to the same value for the temperature of the black hole. This degeneracy translates into discontinuities on the Gibbs function corresponding to transitions from small to large black holes, with the understanding that the horizon radius gives a measure of the size of the black hole.

In this paper, we focused on the equation of state and the Gibbs function of the noncommutative model. We showed that the most direct effect of noncommutativity on classical thermodynamical behavior concerns the existence or absence of critical points. By looking at the Gibbs function $G$, we can determine if a phase transition may exist; it turns out that $\Theta \neq 0$ implies that the Gibbs function becomes smoother and its derivatives with respect to the temperature become single-valued, removing thus critical points; $\Theta$ does not need to take large values for this situation to happen.

Our findings are in agreement with previous results [48] using smeared distributions of matter and charge to include noncommutative effects on classical black hole solutions; in that work, the metric of the noncommutative inspired black hole changes when going from short to large distances. In our treatment, even though perturbative, the behavior of the metric coefficient $\hat{g}_{00}$ also shows changes at short distances, while at large distances it reproduces the standard commutative behavior.

More explicitly, in the noncommutative scenario, the absence of critical points that exist in the commutative case is a direct consequence of the relationship between the temperature $T$ and the Gibbs function $G$ of the noncommutative black hole with its horizon radius. As Fig. 4 shows, there is a degeneracy for both $T$ and $G$ in the commutative case; the noncommutative parameter removes these degeneracies partially in such way that, for example, if for some values of the parameters of the black hole there are three horizons associated to a single value of $G$ in the classical situation, there exists only two for the same $G$ in the noncommutative case.

Finally, in [49], a formalism was put forward to explore in more detail the nature of phase transitions and to analyze features of holographic superconductors; it would be interesting to apply this technique to noncommutative inspired black holes solutions to gain more information on the influence of the noncommutative parameter on the thermodynamical properties of black holes.

\section{ACKNOWLEDGMENTS}

We want to thank the referee for their valuable comments and suggestions to improve our work. R. L. acknowledges partial support from CONACyT Grant No 237351 "Implicaciones físicas de la estructura del espacio-tiempo". O. S-S. acknowledges support from a postdoctoral fellowship of the Programa para el Desarrollo Profesional Docente (PRODEP) Grant No 12312260.

\section{APPENDIX: TEMPERATURE AND EQUATION OF STATE OF THE ADSEBI BLACK HOLE}

For convenience, we provide here the full expression for the temperature and the equation of state of the noncommutative AdSEBI black hole. Up to second order on $\Theta$, they are 


$$
\begin{aligned}
T_{H}=\left.\frac{1}{4 \pi} \frac{d \hat{g}_{00}}{d r}\right|_{r_{H}}= & \frac{1}{4 \pi r_{H}}\left[1+\frac{2 r_{H}^{2}}{b^{2}}-\Lambda r_{H}^{2}+\frac{2 r_{H}^{2}}{b^{2}}\left(1-\sqrt{1+\frac{b^{2} Q^{2}}{r_{H}^{4}}}\right)\right] \\
& +\frac{1}{4 \pi}\left[-\frac{1}{32}\left\{\frac{4}{r_{H}}\left(\frac{2 Q^{2}}{\sqrt{r_{H}^{4}+b^{2} Q^{2}}}-1\right)\left(\frac{1}{r_{H}}+\frac{2 r_{H}}{b^{2}}\left(1-\sqrt{1+\frac{b^{2} Q^{2}}{r_{H}^{4}}}\right)-\Lambda r_{H}\right)\right.\right. \\
& \left.+\left(\frac{1}{r_{H}}-\Lambda r_{H}+\frac{2 r_{H}}{b^{2}}\left(1-\sqrt{1+\frac{b^{2} Q^{2}}{r_{H}^{4}}}\right)\right)^{2}\right\}+\frac{1}{16}\left\{\frac{8}{r_{H}^{3}}\left(\frac{2 Q^{2}}{\sqrt{r_{H}^{4}+b^{2} Q^{2}}}-1\right)^{2}\right. \\
& +4\left(\frac{2 Q^{2}}{\sqrt{r_{H}^{4}+b^{2} Q^{2}}}-1\right)\left(\frac{1}{r_{H}}+\frac{2 r_{H}}{b^{2}}\left(1-\sqrt{1+\frac{b^{2} Q^{2}}{r_{H}^{4}}}\right)-\Lambda r_{H}\right) \\
& +2 r_{H}\left(\frac{6}{r_{H}^{3}}+\frac{4}{b^{2} r_{H}}-\frac{2 \Lambda}{r_{H}}-\frac{4\left(3 b^{4} Q^{4}+6 b^{2} Q^{2} r_{H}^{4}+r_{H}^{8}\right)}{b^{2} r_{H}^{3}\left(r_{H}^{4}+b^{2} Q^{2}\right)^{3 / 2}}\right) \\
& \times\left(\frac{1}{r_{H}}+\frac{2 r_{H}}{b^{2}}\left(1-\sqrt{1+\frac{b^{2} Q^{2}}{r_{H}^{4}}}\right)-\Lambda r_{H}\right)+2\left(\frac{1}{r_{H}}+\frac{2 r_{H}}{b^{2}}\left(1-\sqrt{1+\frac{b^{2} Q^{2}}{r_{H}^{4}}}\right)-\Lambda r_{H}\right) \\
& \left.\left.\times\left(\frac{4}{r_{H}^{2}}+\frac{4}{b^{2}}-2 \Lambda-\frac{4\left(2 b^{4} Q^{4}+5 b^{2} Q^{2} r_{H}^{4}+r_{H}^{8}\right)}{b^{2} r_{H}^{2}\left(r_{H}^{4}+b^{2} Q^{2}\right)^{3 / 2}}\right)\right\}\right] \Theta^{2}+\mathcal{O}\left(\Theta^{4}\right)
\end{aligned}
$$

and

$$
\begin{aligned}
P= & \frac{T_{H}}{v}-\frac{1}{2 \pi v^{2}}+\frac{b^{2}}{4 \pi}\left(1-\sqrt{1+\frac{16 b^{2} Q^{2}}{v^{4}}}\right)+\frac{1}{8 \pi v}\left[\frac { 1 } { 3 2 } \left\{v\left(-\frac{8}{v^{2}}+\frac{64 Q^{2}}{v^{2} \sqrt{v^{4}+16 b^{2} Q^{2}}}\right)\right.\right. \\
& \times\left(4 \pi T_{H}+\frac{v}{b^{2}}\left(1-\frac{v}{b^{2}} \sqrt{1+\frac{16 b^{2} Q^{2}}{v^{4}}}\right)-b^{2} v\left(1-\frac{v}{b^{2}} \sqrt{1+\frac{16 b^{2} Q^{2}}{v^{4}}}\right)\right) \\
& \left.+\left(4 \pi T_{H}+\frac{v}{b^{2}}\left(1-\frac{v}{b^{2}} \sqrt{1+\frac{16 b^{2} Q^{2}}{v^{4}}}\right)-b^{2} v\left(1-\frac{v}{b^{2}} \sqrt{1+\frac{16 b^{2} Q^{2}}{v^{4}}}\right)\right)^{2}\right\} \\
& -\frac{1}{16}\left\{v\left(-\frac{8}{v^{2}}+\frac{64 Q^{2}}{v^{2} \sqrt{v^{4}+16 b^{2} Q^{2}}}\right)^{2}+4\left(-\frac{8}{v^{2}}+\frac{64 Q^{2}}{v^{2} \sqrt{v^{4}+16 b^{2} Q^{2}}}\right)\right. \\
& \times\left(4 \pi T_{H}+\frac{v}{b^{2}}\left(1-\frac{v}{b^{2}} \sqrt{1+\frac{16 b^{2} Q^{2}}{v^{4}}}\right)-b^{2} v\left(1-\frac{v}{b^{2}} \sqrt{1+\frac{16 b^{2} Q^{2}}{v^{4}}}\right)\right) \\
& +2\left(4 \pi T_{H}+\frac{v}{b^{2}}\left(1-\frac{v}{b^{2}} \sqrt{1+\frac{16 b^{2} Q^{2}}{v^{4}}}\right)-b^{2} v\left(1-\frac{v}{b^{2}} \sqrt{1+\frac{16 b^{2} Q^{2}}{v^{4}}}\right)\right) \\
& \times\left(\frac{4}{b^{2}}+\frac{4}{v^{2}}+\frac{16 \pi T}{v}+\frac{v}{b^{2}}-4 b^{2}\left(1-\frac{v}{b^{2}} \sqrt{1+\frac{16 b^{2} Q^{2}}{v^{4}}}\right)-\frac{4\left(512 b^{4} Q^{4}+80 b^{2} Q^{2} v^{4}+v^{8}\right)}{b^{2} v^{2}\left(v^{4}+16 b^{2} Q^{2}\right)^{3 / 2}}\right) \\
& \left.\times\left(\frac{8}{b^{2} v}+\frac{32}{v^{3}}+\frac{32 \pi T}{v^{2}}-\frac{8 b^{2}}{v}\left(1-\frac{v}{b^{2}} \sqrt{1+\frac{16 b^{2} Q^{2}}{v^{4}}}\right)-\frac{8\left(768 b^{4} Q^{4}+96 b^{2} Q^{2} v^{4}+v^{8}\right)}{b^{2} v^{3}\left(v^{4}+16 b^{2} Q^{2}\right)^{3 / 2}}\right)\right\} \Theta^{2}+\mathcal{O}\left(\Theta^{4}\right)
\end{aligned}
$$

respectively. 
[1] N. Seiberg and E. Witten, J. High Energy Phys. 09 (1999) 032.

[2] L. Moller, J. High Energy Phys. 10 (2004) 063.

[3] A. Alboteanu, T. Ohl, and R. Ruckl, Phys. Rev. D 76, 105018 (2007).

[4] R. Horvat, A. Ilakovac, P. Schupp, J. Trampetic, and J.-Y. You, Phys. Lett. B 715, 340 (2012).

[5] P. K. Das and A. Prakash, Int. J. Mod. Phys. A 28, 1350004 (2013).

[6] J. Selvaganapathy, P. K. Das, and P. Konar, Phys. Rev. D 93, 116003 (2016).

[7] F. Ferrari, M. Moskovic, and A. Rovai, Nucl. Phys. B872, 184 (2013).

[8] A. Stern, Phys. Rev. D 89, 104051 (2014).

[9] T. Matsumoto and K. Yoshida, J. High Energy Phys. 06 (2014) 163.

[10] C. D. A. Blair, J. High Energy Phys. 06 (2015) 091.

[11] S. Walters, Nucl. Phys. B894, 496 (2015).

[12] S. Pramanik and S. Ghosh, Gen. Relativ. Gravit. 51, 7 (2019).

[13] M. Maceda and S. Patiño-López, Int. J. Mod. Phys. D (2019), https://doi.org/10.1142/S0218271820500030.

[14] R. H. Brandenberger, Prog. Theor. Phys. Suppl. 171, 121 (2007).

[15] J. Madore, in International Workshop Mathematical PhysicsToday, Priority Technologies-for Tomorrow (1997), https:// www.esi.ac.at/static/esiprpr/esi478.pdf.

[16] J. Madore, in Classical and Quantum Nonlocality. International School of Cosmology and Gravitation XVI Course (World Scientific, Singapore, 2000).

[17] J. W. Moffat, Phys. Lett. B 491, 345 (2000).

[18] J. W. Moffat, Phys. Lett. B 493, 142 (2000).

[19] P. Aschieri, C. Blohmann, M. Dimitrijević, F. Meyer, P. Schupp, and J. Wess, Classical Quantum Gravity 22, 3511 (2005).

[20] P. Aschieri, M. Dimitrijevic, F. Meyer, and J. Wess, Classical Quantum Gravity 23, 1883 (2006).

[21] F. Muller-Hoissen, AIP Conf. Proc. 977, 12 (2008).

[22] L. O. Pimentel and C. Mora, Gen. Relativ. Gravit. 37, 817 (2005).

[23] L. O. Pimentel and O. Obregón, Gen. Relativ. Gravit. 38, 553 (2006).

[24] T. Ohl and A. Schenkel, J. High Energy Phys. 10 (2009) 052.

[25] G. Dvali, C. Gomez, and S. Mukhanov, J. High Energy Phys. 02 (2011) 012.
[26] E. Spallucci and A. Smailagic, in Advances in Black Holes Research, edited by A. Barton (Nova Science Publishers, New York, 2014).

[27] Y. M. Cho, J. Math. Phys. (N.Y.) 16, 2029 (1975).

[28] L. N. Chang, K. I. Macrae, and F. Mansouri, Phys. Rev. D 13, 235 (1976).

[29] F. Mansouri and L. N. Chang, Phys. Rev. D 13, 3192 (1976).

[30] S. W. MacDowell and F. Mansouri, Phys. Rev. Lett. 38, 739 (1977); 38, 1376(E) (1977).

[31] G. Zet, V. Manta, and S. Babeti, Int. J. Mod. Phys. C 14, 41 (2003).

[32] M. Chaichian, A. Tureanu, and G. Zet, Phys. Lett. B 660, 573 (2008).

[33] M. Chaichian, M. R. Setare, A. Tureanu, and G. Zet, J. High Energy Phys. 04 (2008) 064.

[34] G. Zet, Romanian reports in Physics 61, 437 (2009), http:// www.rrp.infim.ro/2009_61_3.html.

[35] M. Born, Proc. R. Soc. A 143, 410 (1934).

[36] M. Born and L. Infeld, Proc. R. Soc. A 144, 425 (1934).

[37] A. García D., H. Salazar I., and J. F. Plebański, Il Nuovo Cimento B 84, 65 (1984).

[38] M. Blagojevic, Gravitation and Gauge Symmetries (IOP, Bristol, 2002), p. 522.

[39] Gauge Theories of Gravitation, edited by M. Blagojević and F. W. Hehl (World Scientific, Singapore, 2013).

[40] G. Zet, C. D. Oprisan, and S. Babeti, Int. J. Mod. Phys. C 15, 1031 (2004).

[41] G. Zet, C. Popa, and D. Partenie, Commun. Theor. Phys. 47, 843 (2007).

[42] S. Babeti, in Lie Theory and Its Applications in Physics, edited by V. Dobrev, Springer Proceedings in Mathematics \& Statistics Vol. 111 (Springer, Tokyo, 2014), pp. 231-239.

[43] H. J. Groenewold, Physica (Utrecht) 12, 405 (1946).

[44] J. E. Moyal, Proc. Cambridge Philos. Soc. 45, 99 (1949).

[45] A. H. Chamseddine, Phys. Lett. B 504, 33 (2001).

[46] K. Ülker and B. Yapişkan, Phys. Rev. D 77, 065006 (2008).

[47] K. Ülker, Int. J. Mod. Phys. Conf. Ser. 13, 191 (2012).

[48] A. González, R. Linares, M. Maceda, and O. SánchezSantos, Int. J. Theor. Phys. 57, 2041 (2018).

[49] D. Roychowdhury, Ph.D. thesis, Bose National Center, 2013. 